\title{
Posterior dynamic stabilization of the lumbar spine: pedicle- based stabilization with the AccuFlex rod system
}

\author{
Christopher E. Mandigo, M.D., ${ }^{1}$ Prakash Sampath, M.D., ${ }^{2}$ \\ AND MiChael G. KAISER, M.D. ${ }^{1}$ \\ ${ }^{1}$ Department of Neurological Surgery, Columbia University College of Physicians and Surgeons, \\ New York, New York; and ${ }^{2}$ Department of Neurosurgery, Brown Medical School, \\ Providence, Rhode Island
}

\begin{abstract}
$\checkmark$ Posterior dynamic stabilization in the lumbar spine is performed in an attempt to reduce loading across the intervertebral disc for the purpose of relieving pain and limiting degeneration while preserving motion. The AccuFlex rod system (Globus Medical, Inc.), a first-generation device, achieves this by changing the properties of the rod within the Protex pedicle screw-based rigid rod system. Helical cuts that have been created in the standard 6.5-mm rod allow for a limited range of motion while providing a posterior tension band that relieves a significant amount of disc loading. The AccuFlex rod system has been approved by the Food and Drug Administration for single-level fusion when used in conjunction with an interbody graft. In a study involving 170 patients who underwent fusion surgery for back pain, the 54 who received the AccuFlex construct had statistically similar fusion rates and outcomes (as assessed by visual analog scale and Short Form-16 scores) when compared with 116 patients treated with rigid rod fixation after 1 year of follow up. Future clinical studies will examine and provide information regarding the impact of AccuFlex on the incidence of adjacent-level disease. Information gained through the clinical experience with AccuFlex will serve as a foundation for the development of a stand-alone dynamic construct.
\end{abstract}

KEY WORDS • posterior dynamic stabilization $\bullet$ lumbar spine $\quad$ spinal fusion

$\mathrm{S}$ URGICAL management of mechanical low-back pain has primarily been directed toward alleviating pain caused by instability or abnormal load distribution on the lumbar spine. Stabilization of abnormal or degenerated lumbar segments has usually been achieved through fusion surgery, commonly supplemented by rigid pedicle screw fixation and, more recently, by interbody grafting. Recent clinical studies have demonstrated that despite improved rates of lumbar fusion with newer techniques, there has been no concomitant increase in pain relief. ${ }^{1}$ Many patients also fail to improve despite successful fusion. These observations, coupled with biomechanical investigations of normal and degenerated lumbar discs, have created a more comprehensive understanding of the possible mechanisms that contribute to mechanical low-back pain.

Abnormal load transmission across a degenerated lumbar disc is a possible mechanism of accelerated degeneration and pain. This pattern has been recognized in other joints as the primary pain generator contributing to symptomatic osteoarthritis ${ }^{6,7}$ It is also consistent with the typical clinical setting for low-back pain, in which pain is more related to position and posture than to abnormal lumbar spine movement. ${ }^{5}$

Dynamic stabilization is performed in an attempt to change the biomechanics of the affected lumbar segment by reducing the load on the disc without the disadvantages that can result from the loss of motion created by fusion. A dynamic system would work in this context by limiting motion and altering stress patterns across the degenerated segment, preventing excessive motion or postures that result in pain. The theoretical appeal of this approach is that it may avoid the potential negative effects on the adjacent segment and on the overall biomechanics of the spine that result from fusion. The concept of dynamic stabilization may also provide an advantage over rigid fixation when used as a posterior tension band supplement in lumbar fusion surgery. Through Wolff's law, the transmission of forces to an intervertebral graft and avoidance of "stress shielding" could potentially increase the rate and likelihood of a successful arthrodesis. ${ }^{2}$

The practice of dynamic lumbar stabilization has existed for approximately a decade in Europe. The constructs with the most extensive use, such as the Graf ligament system and the Dynesys system, involve pedicle screws joined across lumbar segments with nonelastic bands. ${ }^{3,4}$ With these constructs, surgeons attempt to create a posterior tension band to place the motion segment in extension and to limit movement in other directions. An important element of this technique is the preservation of the posterior ligaments and facet joints during surgical exposure. Clear contraindications are osteoporotic bone and evidence of severe segmental instability (that is, gross spondylolisthesis). The initial results with these devices have proven to be encouraging, with results comparable to those seen with fusion. The AccuFlex system (Globus Medical, Inc.) is a pedicle screw/rod-based construct that incorporates the insertional techniques of current rigid fix- 


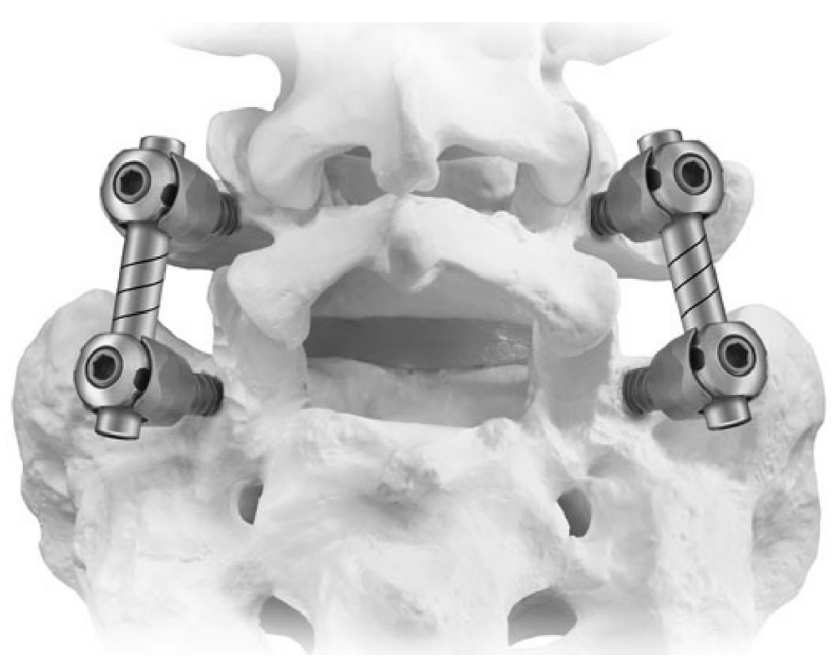

FIG. 1. Photograph of a spine model showing the proper placement of the AccuFlex construct at the L5-S1 position.

ation technology, with the potential benefits of dynamic stabilization.

\section{The AccuFlex Construct}

AccuFlex is a semirigid rod intended for use with the Protex (Globus Medical, Inc.) 6.5-mm pedicle screwbased rod. At present, AccuFlex is approved by the Food and Drug Administration for lumbar fusions when used in conjunction with an anterior interbody device (Fig. 1). This novel construct is created by a double helical cut made within a standard $6.5-\mathrm{mm}$ rod. The cut creates flexibility, transforming a rigid rod into a semirigid one that allows motion primarily in the flexion-extension mode. Despite designation as a soft-stabilization device, the technique for AccuFlex implantation is nearly identical to that used for the standard pedicle screw/rod construct, the only exception being a more precise positioning of the rod between the pedicle screws. Due to these similarities, the insertion of an AccuFlex construct can be readily performed by most spine surgeons and is easily converted to a rigid construct simply by replacing the rod, should revision be required. AccuFlex is the first stage in the development of a stand-alone dynamic stabilization construct.

\section{Construct Design}

The AccuFlex construct is intended to limit axial rotation and lateral bending while permitting functional flexion and extension movements. It also provides compressive stiffness to unload the intervertebral disc and the facet joints across a single spinal segment. The intended flexion and extension movements increase the load transmitted through an anterior interbody graft by more than 50\% compared with a rigid construct, creating a "load-sharing" environment as opposed to "stress shielding." This loadsharing capability of the AccuFlex construct is intended to increase the potential for fusion through Wolff's law. ${ }^{2}$

The AccuFlex rod is created using a proprietary machining process known as electrical discharge machining. In this technique a conductive wire is used which, when connected to a power supply, creates a potential difference between itself and the work piece. This produces an arc that erodes the material as the wire is moved along a specified path that is under computerized, numerical control. The double helical cut runs through the center of the rod and generates limited degrees of flexibility. The amount of flexibility depends on the number of circumferential cuts made within a specified length of rod; the more spiral cuts there are, the more flexible the rod (Fig. 2). A single AccuFlex rod is currently available with a $540^{\circ}$ helix. The remaining components of the construct are identical to a typical pedicle screw-based system. The Protex system is a top-loading, multiaxial pedicle screw-based construct that provides $30^{\circ}$ of angulation. The locking mechanism is a nonthreaded design intended to eliminate cross-threading and allows for rod adjustment prior to final tightening of the center setscrew.

Determining the appropriate degree of flexibility is one limitation of the AccuFlex rod, as well as other dynamic constructs, because this question remains unanswered. The degree of flexibility is not adjustable. Characteristics that limit the use of the AccuFlex across a single lumbar segment include the inability to adjust the position of the flexible rod component to account for variable pedicle screw placement and the inability to contour the rod. Therefore, the AccuFlex construct has been limited to the treatment of single-level disease.

\section{Biomechanical Testing}

The AccuFlex rod has undergone extensive biomechanical testing to prove its safety as a posterior stabilization construct. Testing has been performed in multiple modes including compression/tension, axial rotation, sagittal rotation, compression, and pure shear (Table 1). The results of these tests have demonstrated that the rods can withstand the normal stresses exerted on the lumbar spine with an adequate fatigue life ( $>10$ million stress movements without fracture) to prevent failure prior to fusion formation.

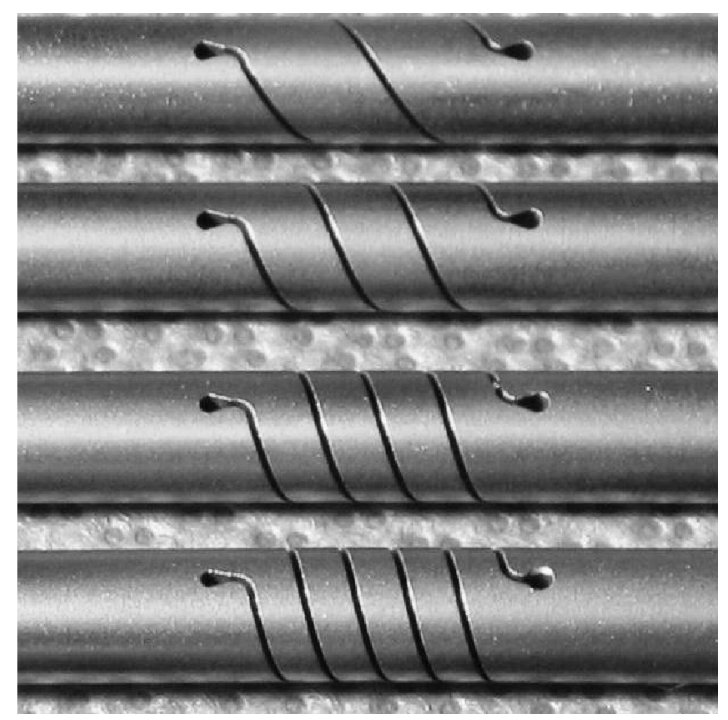

FIG. 2. Photograph showing a series of AccuFlex rods with increasing numbers of spiral cuts: the rod at the bottom of the picture is significantly more flexible than the top rod. Currently, only one type of AccuFlex rod is available. It has a $540^{\circ}$ helical cut. 
TABLE 1

Fatigue testing performed to evaluate the AccuFlex rod*

\begin{tabular}{ll}
\hline \multicolumn{1}{c}{ Motion } & \multicolumn{1}{c}{ Conditions Tested } \\
\hline load bearing & $\pm 100 \mathrm{~N} ; 10^{7}$ cycles \\
& $+100 \mathrm{~N} /+800 \mathrm{~N} ; 10^{7}$ cycles \\
pure shear & $\pm 140 \mathrm{~N} ; 10^{7}$ cycles \\
compression shear & $\pm 100 \mathrm{~N} ; 10^{7}$ cycles \\
rotation & $\pm 2^{\circ} \mathrm{ROM} ; 10^{7}$ cycles \\
tension/compression & $\pm 500 \mathrm{~N} ; 10^{7}$ cycles \\
compression bend & $\pm 2 \mathrm{~mm} ; 10^{7}$ cycles \\
\hline
\end{tabular}

*ROM $=$ range of motion.

\section{Implantation Technique}

The implantation of an AccuFlex construct is nearly identical to that performed for the typical pedicle screwbased stabilization system. Once the pedicle screws are placed, the rod is positioned between them with the helical cut placed equidistant from each screw. It is essential that the locking screws not engage the rod at the helical cut, because this could create a significant stress riser that increases the potential for rod fracture. At the terminal ends of the helical cut, a hole is bored into the rod. These holes must be oriented in a medial-to-lateral direction to allow for flexibility in the sagittal plane. Figure 3 is an intraoperative photograph of the properly placed construct, and Fig. 4 is a lateral x-ray film of the construct in place at L4-5 with an interbody graft. In the event that a revision is required or more rigid stabilization desired, the AccuFlex is removed by disengaging the locking screws, and it can then be replaced with a rigid rod.

\section{Clinical Examples}

The use of the AccuFlex system has been evaluated in a prospective, randomized study of patients receiving fusion for mechanical low-back pain (unpublished data). The patients were randomized to receive either the AccuFlex or a standard $6.5-\mathrm{mm}$ rigid rod combined with interbody grafting at one lumbar level. The end points examined were fusion, rates of fusion, and the visual analog scale and Short Form-16 pain score outcome measures over 1 year of follow up. In this study, 54 patients received

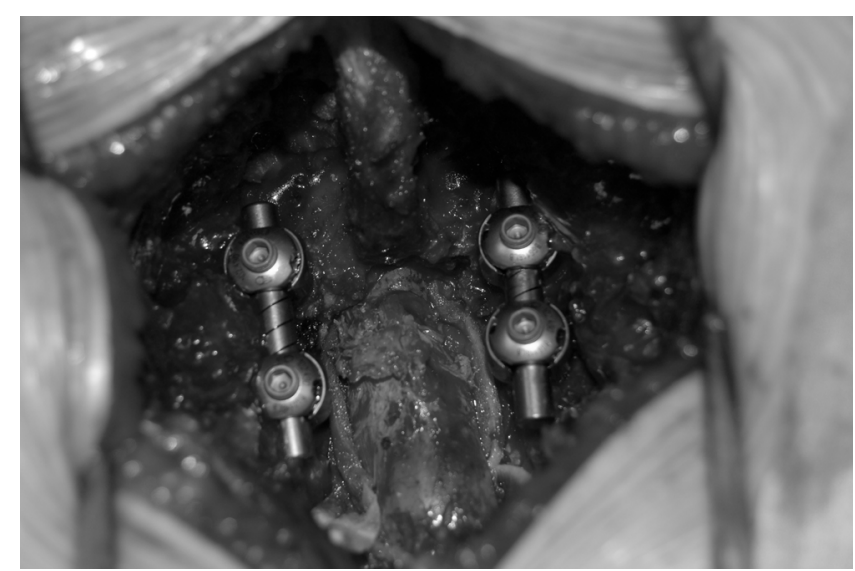

FIG. 3. Intraoperative photograph of the properly placed construct.

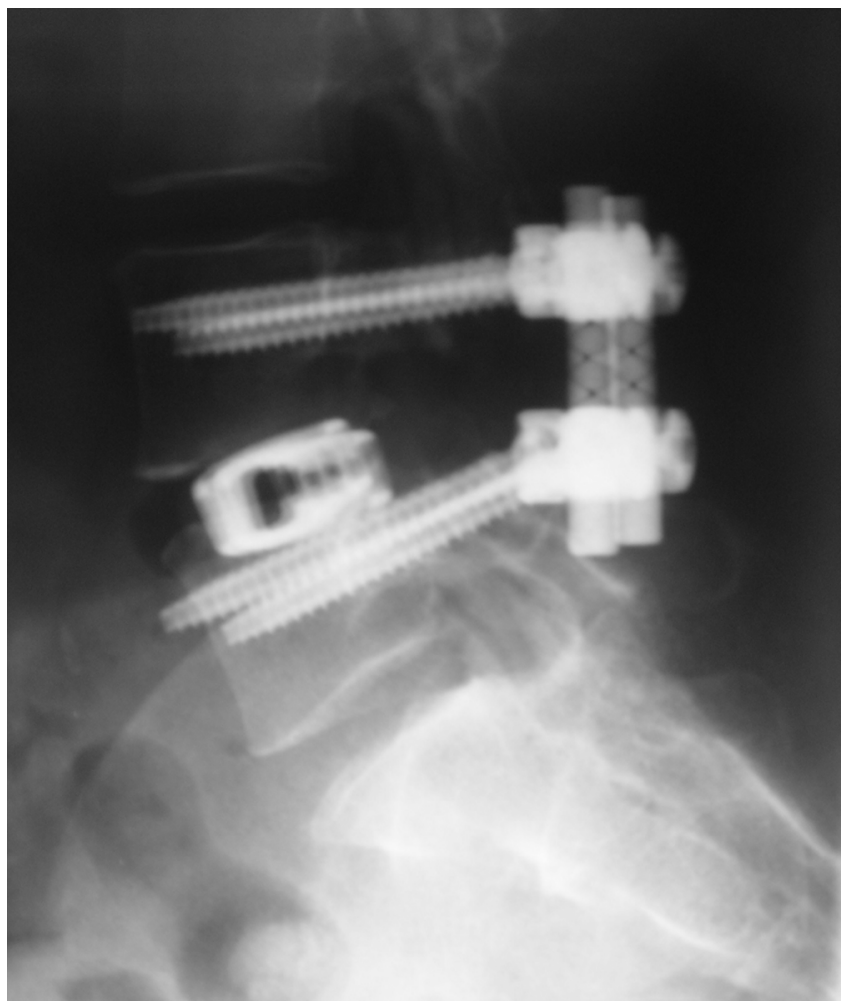

FIG. 4. Lateral $x$-ray film showing the AccuFlex construct at the L4-5 level, along with an interbody graft.

the AccuFlex rod with interbody grafting (46 transforaminal lumbar interbody fusion, seven posterior lumbar interbody fusion, and one anterior lumbar interbody fusion) and 116 patients received the standard rigid rod with interbody grafting (101 transforaminal lumbar interbody fusion and 15 posterior lumbar interbody fusion). Posterior-lateral fusion was performed with a combination of auto- and allografting. There were no instrumentation failures in either group, and there was a nonsignificant trend toward faster fusion in the AccuFlex group. The visual analog scale and Short Form-16 scores were not significantly different over the course of the study, and the fusion rate was not significantly different between the two groups. After 1 year, the AccuFlex group fusion rate was $92 \%$ (50 of 54 patients) and the rate in the standard rigid rod group was $95 \%$ (110 of 116 patients.)

\section{Conclusions}

Currently, the AccuFlex construct is approved for single-level fusion when used in conjunction with an anterior interbody graft. Given the characteristics that limit its use to a single level and the inability to modify the degree of flexibility postmanufacturing, it is not intended as a nonfusion stabilization device. AccuFlex represents the first stage in the evolution of a soft-stabilization device. Whether the inclusion of such a device more closely mimics a noninstrumented fusion and decreases the degree of adjacent-level stress has not been proven. Studies are currently being conducted to determine if this is indeed the case; however, the clinical data required to prove this hypothesis will take years to accumulate. 
The evolution of the AccuFlex does highlight the difficulty in designing a stand-alone posterior dynamic lumbar device. Future generations of this construct are now in the design stage. An essential question that remains unanswered is the degree of stabilization that a nonfusion construct should provide. Not all pathological processes result in the same degree or type of instability; therefore, it is possible that there is no single solution, and multiple devices may evolve to treat different pathological processes.

\section{Disclosure}

Dr. Kaiser serves on the strategic advisory panel for Globus Medical, Inc. Dr. Kaiser has no personal financial interest in Globus Medical. Funds received for his participation are placed in an unrestricted research account. Dr. Sampath serves on the strategic advisory panel for Globus Medical and is a shareholder in the company.

\section{References}

1. Boos N, Webb JK: Pedicle screw fixation in spinal disorders: a European view. Eur Spine J 6:2-18, 1997

2. Frost HM: A 2003 update of bone physiology and Wolff's law for clinicians. Angle Orthod 74:3-15, 2004
3. Gardner A, Pande KC: Graf ligamentoplasty: a 7-year followup. Eur Spine J 11 (2 Suppl):S157-S163, 2002

4. Grob D, Benini A, Junge A, Mannion AF: Clinical experience with the Dynesys semirigid fixation system for the lumbar spine: surgical and patient-oriented outcome in 50 cases after an average of 2 years. Spine 30:324-331, 2005

5. Smith D, McMurray N, Disler P: Early intervention for acute back injury: can we finally develop an evidence-based approach? Clin Rehabil 16:1-11, 2002

6. Troum OM, Crues JV III: The young adult with hip pain: diagnosis and medical treatment, circa 2004. Clin Orthop Relat Res 418:9-17, 2004

7. Yamamoto I, Panjabi MM, Crisco T, Oxland T: Three-dimensional movements of the whole lumbar spine and lumbosacral joint. Spine 14:1256-1260, 1989

Manuscript submitted October 20, 2006.

Accepted November 20, 2006.

Address reprint requests to: Michael G. Kaiser, M.D, Department of Neurological Surgery, Neurological Institute, Spine Center-5th floor, 710 West 168th Street, New York, New York 10032.email: mgk7@columbia.edu. 